\title{
Qubits meet materials science
}

\author{
Qubits come in many shapes and forms. Some are better developed, some will make it easier to \\ scale up to big quantum processors and some will require less effort to correct errors. One thing \\ they have in common: they will all benefit from materials optimization.
}

Earlier this summer, a group of Chinese researchers led by Jian-Wei Pan announced the most powerful quantum computer currently available. The machine, which comprises 66 superconducting qubits, outperformed the 53-qubit quantum computer Google used to claim quantum advantage in 2019.

Alongside superconducting qubits, several qubit platforms are being developed, each with its own advantages and drawbacks: the jury is still out regarding which one will power the first practical quantum computer. Much progress so far has been achieved through device optimization, but, moving forwards, improvements in materials quality and fabrication will be important to fuel further progress. In this focus issue, we explore the main materials-related challenges that need to be addressed for the different types of qubit.

Qubits leverage two quantum effects - superposition and entanglement - to outdo classical bits, the binary digits that constitute the basic units of information in computing. A qubit can be in the ' 0 ' and ' 1 ' state simultaneously and can share its state with other qubits in such a way that the system's processing capability doubles with every qubit. Thus, a quantum computer comprising seven entangled qubits can support $128\left(2^{7}\right)$ computations at the same time, rather than having to perform them sequentially as a classical computer would. Although a quantum computer will not outperform a classical computer for any given task, it will provide a dramatic advantage for tasks that require exploring many different paths simultaneously, such as searches in large datasets or quantum-chemical computations.

Although not yet peer-reviewed, the result from Pan's group confirms the leading position superconducting qubits hold in the quantum computing race. These qubits are based on superconducting circuits that can be switched between two energy levels, the ' 0 ' and ' 1 ' states of the qubit, with microwave pulses. Superconducting qubits have the advantage that they can be fabricated using techniques from conventional computer chip manufacturing and operate very quickly. However, as Irfan Siddiqi discusses in a Review, their computational power is still limited by non-equilibrium excitations and by the decoherence that results from materials defects; thus, materials optimization will be important to obtain further progress.

Qubits based on ion traps are also leading contenders for a practical quantum computer. These qubits are based on the internal electronic states of atomic ions, which are held in position by electric fields and can be manipulated using optical and microwave signals. Trapped-ion qubits can have extremely long coherence times of up to 6,000s, and universal ion-trap quantum computers with up to 11 ions have been demonstrated. Although at first sight trapped-ion quantum processors do not involve a lot of materials science, materials optimization is essential to design traps that efficiently integrate optical and electronic components for qubit control while minimizing the electric-field noise that plagues these qubits, as Kenneth Brown and colleagues discuss in a Review.

The ability to fabricate many identical qubits will be essential to build a working quantum computer. Hence, qubits that can leverage the mature manufacturing infrastructure of the semiconducting industry - qubits that store information in the spin of quantum dots in semiconductors, particularly silicon - are also attracting substantial attention, despite being less well developed than superconducting and trapped-ion qubits. In general, the interplay between the spin, optical and charge states of solid-state spin defects can be exploited to realize complex experiments and technologies, as David Awschalom and colleagues discuss in a Review, and qubits based on quantum dots have the advantageous properties of long coherence times and high working temperatures. An emerging platform in this context, as Giordano Scappucci and colleagues discuss in a Review, are germanium qubits, for which a four-qubit quantum processor has been recently demonstrated.

Finally, there are qubits that are highly promising, but do not yet exist. Topological qubits are based on quasiparticles whose quantum states are encoded in the braiding paths they follow in time. Because the quantum information stored this way is topologically protected, this type of qubit would not require complex error correction protocols. Although there is evidence that the quasiparticles themselves - Majorana quasiparticles have been realized, their braiding has so far been elusive. The different materials systems in which Majorana quasiparticles are being explored are the subject of a Review by Ady Stern and colleagues.

The quantum race is on, and, as materials optimization takes on a more prominent role, the contribution of materials scientists will be important to develop each type of qubit and to determine what practical quantum computers will look like. 